\title{
The role of absolute and relative amounts of time in forgetting within immediate memory: The case of tone-pitch comparisons
}

\author{
NELSON COWAN, J. SCOTT SAULTS, and LARA D. NUGENT \\ University of Missouri, Columbia, Missouri
}

\begin{abstract}
Many investigators of auditory sensory memory have assumed that memory loss during a retention interval of some seconds results from a process (such as decay) that depends on the absolute amount of time that has elapsed since presentation of the sound. An alternative possibility, brought to light by studies on immediate verbal memory, is that it is the relative, rather than the absolute, amount of time that matters. We examine these factors in a tone-comparison study by varying not only the retention interval between two tones to be compared, but also the interpair interval. Relative time played a role, but absolute time also appeared to be important. Several concepts of "decay" are considered in relation to the results.
\end{abstract}

This study reassesses auditory memory loss in light of a recent theoretical debate about the nature of forgetting in immediate memory. At least two kinds of memory limitation can be proposed. The first is a limit to how long an item can be held in memory. Some memory representations may "decay," or become weaker or less complete, as a function of the passage of time (e.g., Broadbent, 1958). The second is a limit to how many items can be held in memory at one time (e.g., Miller, 1956). Given an item limit, loss of an item is attributed to interference from other items.

The interference principle is so well established that perhaps no theorist denies its existence. Baddeley (1986) proposed that phonological memory was lost within about $2 \mathrm{sec}$ unless it was rehearsed, with no mention of interference; but that may have been intended as a simplifying assumption. We believe that most decay theorists suppose that both decay and interference take place concurrently. However, a pure interference theorist holds that no true decay occurs, only interference. The existence or nonexistence of decay in immediate memory has been debated for a long time (e.g., Bjork \& Whitten, 1974; Broadbent, 1958; Brown \& Hulme, 1995; Cowan, 1988, 1995; Cowan et al., 1992; Cowan, Wood, Nugent, \& Treisman, in press; Crowder, 1976, 1993; Gardiner \& Gregg, 1979; Glenberg \& Swanson, 1986; Keppel \& Underwood, 1962; Koppenaal \& Glanzer, 1990; McGeoch, 1932; Melton, 1963; Nairne, 1992; Neath \& Nairne, 1995; Peterson \& Peterson, 1959; Schweickert \& Boruff, 1986).

A related issue is whether mental events (thoughts, images, etc.) should be counted as mechanisms of inter-

This research was supported by NIH Grant HD-21338 to N.C. Address reprint requests to N. Cowan, Department of Psychology, $210 \mathrm{Mc}$ Alester Hall, University of Missouri, Columbia, MO 65211 (e-mail: psycowan@showme.missouri.edu). ference or decay. Theoretical issues of this sort make it difficult to investigate the question of decay directly. However, a related, better defined issue that can be examined is whether the absolute amount of time in the retention interval makes a difference, as a decay theorist must believe, or whether only the amount of time relative to other events in the stimulus matters, as some interference theorists have suggested (e.g., Crowder, 1993). For verbal immediate memory, many investigators have recognized the precarious status of absolute time as a causal factor. That realization has come partly because the recency effect in free recall can be obtained after a long, list-final distracting task provided that the items are also separated by distracting tasks, which increase temporal distinctiveness (Bjork \& Whitten, 1974). Similarly, the loss of memory of a consonant triad during a distracting task occurs only after several trials have been completed, suggesting that long retention intervals may lower recall not by allowing decay, but by eliminating the temporal distinctiveness of the last triad (Keppel \& Underwood, 1962). These findings do not prove that there is no decay factor operating in such tasks (e.g., see Cowan, 1995), but they challenge the decay concept.

Within the area of auditory sensory memory, many investigators have assumed that there is a role of absolute time. (For a review, see Cowan, 1984.) For example, there have been many studies in which subjects heard two tones separated by a silent retention interval (RI) on a particular trial and were to compare the pitches of the tones. The general finding is that the proportion correct declines as the duration of the RI increases, even when there is no task for the subject between the tones other than to try to remember the first tone. The conventional conclusion from tone-comparison studies is that the auditory sensory memory representation of the first tone in the pair is lost during the RI as a function of the 
passage of time per se, making that representation increasingly unavailable for comparison with the second tone.

However, there is an oversight in the vast soundcomparison literature that challenges the above interpretation. To our knowledge, researchers in that area have attended to only one of two problems with inferences about absolute time. They have tried to control for effects of retroactive interference in memory during the retention interval (e.g., Massaro, 1970). However, they have not worried about the temporal distinctiveness of the sounds within the current trial. This distinctiveness theoretically could play such an important role that it might even account for all of the memory loss over time, with no role of decay at all. One reason is as follows.

In two-tone comparison tasks, the time between the second tone of one trial and the first tone of the next typically ranges from 2 to $6 \mathrm{sec}$, long enough for the subject to make a response. However, performance typically declines until the tones in a pair are separated by an RI of about $20-30 \mathrm{sec}$. Notice that when the RI is very long, the first tone in a pair is closer in time to the tones of the previous trial than it is to the tone to which it is supposed to be compared. If the tones are grouped in memory on the basis of temporal proximity, a long RI could increase the tendency for the two tones in a pair to be perceived as being in different groups, making a comparison between them more difficult.

We carried out an experiment to examine this grouping factor in tone comparisons. The silent RIs between tones to be compared could be $1.5,3.0,6.0$, or 12.0 sec. However, unlike previous studies, the silent time between tone pairs, which we will term the interpair interval (IPI), also was varied independently. The IPI (which included the response to the previous pair and then a silent distracting task) could be $3.0,6.0,12.0$, or $24.0 \mathrm{sec}$. There were 16 conditions in the experiment, formed from each possible combination of length of the prior IPI and length of the RI. All conditions were presented to all subjects.

The mechanism of an overall effect of the RI could be either time-based forgetting or a loss of temporal distinctiveness of the current trial's tones with increasing RIs. It is possible to distinguish between these mechanisms by examining trials with a fixed IPI:RI ratio, for which temporal distinctiveness is, for all practical purposes, held constant. (Any remote effect of the durations between tones two or more trials earlier than the current one is assumed to be negligible.) The intervals that we selected provided four data points for which the RI varied over a wide range $(1.5-12 \mathrm{sec})$, but for which the IPI:RI ratio remained fixed at $2: 1$. It was also possible to examine data points for several other IPI:RI ratios. Any effect of the RI among trials with a common ratio is taken to indicate a loss of memory due to the absolute amount of time that elapsed between tones, rather than temporal distinctiveness.

\section{METHOD}

\section{Subjects}

Subjects ( $N=28 ; 16$ men and 12 women) received course credit for their participation. Data from two other men were excluded because their overall performance levels were near ceiling ( $\geq 97 \%$ correct).

\section{Stimuli}

The sounds were 200 -msec-long sine-wave tones, with on- and offramps of $20 \mathrm{msec}$, played at approximately $63 \mathrm{~dB}(\mathrm{~A})$ as measured with a sound-level meter and earphone coupler. On the basis of pilot data, tone sets were constructed for frequency differences between tones $(\Delta f \mathrm{~s})$ of $1 \%, 1.5 \%, 2 \%$, and $3 \%$. Each of these sets included standard tones with a frequency of $500,625,781,977,1221,1526,1907$, and $2384 \mathrm{~Hz}$. For a particular set of tones with $\Delta f=X \%$, the second, comparison tone was $X \%$ lower or $X \%$ higher in frequency than the standard. For example, within the $2 \%$ tone set, a standard tone of $500 \mathrm{~Hz}$ could be followed by a comparison tone of either 490 or $510 \mathrm{~Hz}$. Each of the eight standard tones could be followed by a higher or a lower tone. The first tone in a trial was preceded by an IPI of $3,6,12$, or $24 \mathrm{sec}$, and the tones were separated by an RI of $1.5,3,6$, or $12 \mathrm{sec}$. All 256 combinations of these possible conditions were used for each subject.

\section{Apparatus and Procedure}

The experiment was administered to each subject individually in a sound-attenuated room via a Power Macintosh computer and TDH-49 audiological headphones. Following a brief familiarization, the first phase of the experiment was a pretest designed to determine the level of $\Delta f$ that the subject could discriminate. The resulting level of $\Delta f$ was used for that subject throughout a brief practice phase and the main phase of the experiment

Familiarization and pretest phases. Subjects learned what the tones would sound like and learned to use the up and down arrow keys on the computer to label each tone pair according to whether the second tone was higher or lower in pitch than the first. To alert the subject on each trial, a change in the background color of the screen from yellow to blue occurred $250 \mathrm{msec}$ before the first tone in a pair was to be presented. Blue was maintained, with no distracting task but with the word "listen" printed on the screen in large type, until after the second tone in the pair was presented. Then a response screen appeared, with a green background. The subject had $2 \mathrm{sec}$ in which to use the arrow keys to respond "higher" or "lower," and a response was followed immediately by feedback for $500 \mathrm{msec}$ indicating whether the response was correct or not. Failure to respond within a 2 -sec period resulted in the response's being counted as incorrect and in feedback stating that.

In the pretest, subjects received tone sets with $3 \%, 2 \%, 1.5 \%$, and $1 \% \Delta f \mathrm{~s}$, in that order. Within each tone set, each of the 16 possible tone pairs occurred once (in random order), always with a 1.5 - sec RI between tones in a trial and a 3-sec IPI between the second tone of one trial and the first tone of the next. The smallest $\Delta f$ at which the subject was correct on 13 of 16 trials was the one used in the main phase of the experiment. However, if a subject did not achieve this level of success for any $\Delta f$, testing proceeded with a $\Delta f$ of $3 \%$. In pilot testing we had found that subjects still performed in the testing phase at a level considerably above chance. The lowest individual mean performance level was $62 \%$.

Practice and test phases. Each subject was tested only at the $\Delta f$ resulting from the pretest. The first three trials were practice trials for which a silent task was introduced. It was to be carried out repeatedly during each IPI (immediately after the response to the prior tone pair, taking up $\leq 2$ sec of the (PI), but not during the RI. The silent task involved pressing the left or right arrow key to signify the direction of movement of a small icon on the screen. The purpose of this silent task was to ensure that the subject remained engaged in the experiment and facing the screen during these sometimes long IPIs. After each left or right arrow keypress in the silent task, there was a 250-msec delay until the icon moved again, 11 to 25 pixels left or right, randomly determined with the restriction that it could not move off the screen. To illustrate to subjects the range of delays, the three practice trials were ran- 
domly selected with several restrictions. Three different tone pairs were used, the longest and shortest RIs and IPIs both were used at least once, and at least one upward and one downward shift in frequency were included in the first two practice trials. The procedure was otherwise the same as in the pretest phase.

In the test phase, each of the 256 possible combinations of tones and timing occurred once. The procedure for these trials was otherwise identical to that used in the practice phase.

\section{RESULTS}

The number of subjects who were tested at each $\Delta f$ and the overall proportion correct achieved by those subjects in the test phase were as follows: for $\Delta f=1 \%, N=$ $5, M=.83, S D=.11$; for $\Delta f=1.5 \%: N=5, M=.86$, $S D=.06 ;$ for $\Delta f=2 \%, N=3, M=.84, S D=.11$; and for $\Delta f=3 \%, N=15, M=.76, S D=.12$.

The means for all combinations of IPI and RI are shown in Table 1. An analysis of variance (ANOVA) with IPI and $\mathrm{RI}$ as factors produced main effects of both IPI $[F(3,81)=$ $\left.3.93, M S_{\mathrm{e}}=0.006, p=.011\right]$ and $\mathrm{RI}[F(3,81)=20.01$, $\left.M S_{\mathrm{e}}=0.010, p=.000\right]$. However, there was no interaction between these factors $(F<1)$. Table 1 shows that performance was best when the IPI was long and when the RI was short.

According to the standard signal-detection-theory analysis of two-interval forced-choice tasks (e.g., Green, 1964), a $d^{\prime}$ sensitivity measure can be calculated from the proportion correct. It is reported within brackets in Table 1, along with numbers showing the discrepancy between each mean proportion and the corresponding proportions for ascending or descending trials only. The fact that all but one of these numbers are positive indicates that performance was generally a little better in ascending-tone trials; this corresponds to a well-known time-order error in comparison judgments (Hellström,

Table 1

Mean Proportion Correct in Each Condition and Associated Measures

\begin{tabular}{|c|c|c|c|c|}
\hline \multirow{2}{*}{$\begin{array}{l}\text { Interpair } \\
\text { Interval }\end{array}$} & \multicolumn{4}{|c|}{ Retention Interval Between Tones in a Pair } \\
\hline & $1.5 \mathrm{sec}$ & $3 \mathrm{sec}$ & $6 \mathrm{sec}$ & $12 \mathrm{sec}$ \\
\hline $3 \mathrm{sec}$ & $\begin{array}{c}.81 \\
{[1.24, .01]}\end{array}$ & $\begin{array}{c}.82 \\
{[1.29, .01]}\end{array}$ & $\begin{array}{c}.79 \\
{[1.14, .03]}\end{array}$ & $\begin{array}{c}.70 \\
{[0.74, .04]}\end{array}$ \\
\hline $6 \mathrm{sec}$ & {$[1.29,-.01]$} & $\begin{array}{c}.83 \\
{[1.34, .04]}\end{array}$ & $\begin{array}{c}.80 \\
{[1.19, .04]}\end{array}$ & $\begin{array}{c}.76 \\
{[1.00, .06]}\end{array}$ \\
\hline $12 \mathrm{sec}$ & $\begin{array}{c}.85 \\
{[1.47, .03]}\end{array}$ & $\begin{array}{c}.82 \\
{[1.29, .02]}\end{array}$ & $\begin{array}{c}.82 \\
{[1.29, .02]}\end{array}$ & $\begin{array}{c}.74 \\
{[0.90, .02]}\end{array}$ \\
\hline $24 \mathrm{sec}$ & $\begin{array}{c}.86 \\
{[1.53, .01]}\end{array}$ & $\begin{array}{c}.82 \\
{[1.29, .02]}\end{array}$ & $\begin{array}{c}.82 \\
{[1.29, .05]}\end{array}$ & $\begin{array}{c}.76 \\
{[1.00, .06]}\end{array}$ \\
\hline
\end{tabular}

Note-The first number reported in brackets is $d^{\prime}$, a sensitivity measure, according to Elliott (1964, Table II), calculated from the group mean. The second number in brackets, when added to (or subtracted from) the proportion correct, yields the proportion correct for ascending (or descending) tone trials only. The standard errors of the mean for individual cells range from .02 to .03 . Based on the subjects $\times$ conditions $M S_{\mathrm{e}}$ from a one-way ANOVA across all conditions, a within-subjects $95 \%$ confidence interval for each cell (see Loftus \& Masson, 1994) is $M \pm .03$. These confidence intervals characterize the pattern of means by condition. However, the $95 \%$ confidence interval for the grand mean is $.80 \pm .04$, and the entire pattern by conditions has to be shifted up or down within that interval to yield the correct population grand mean.

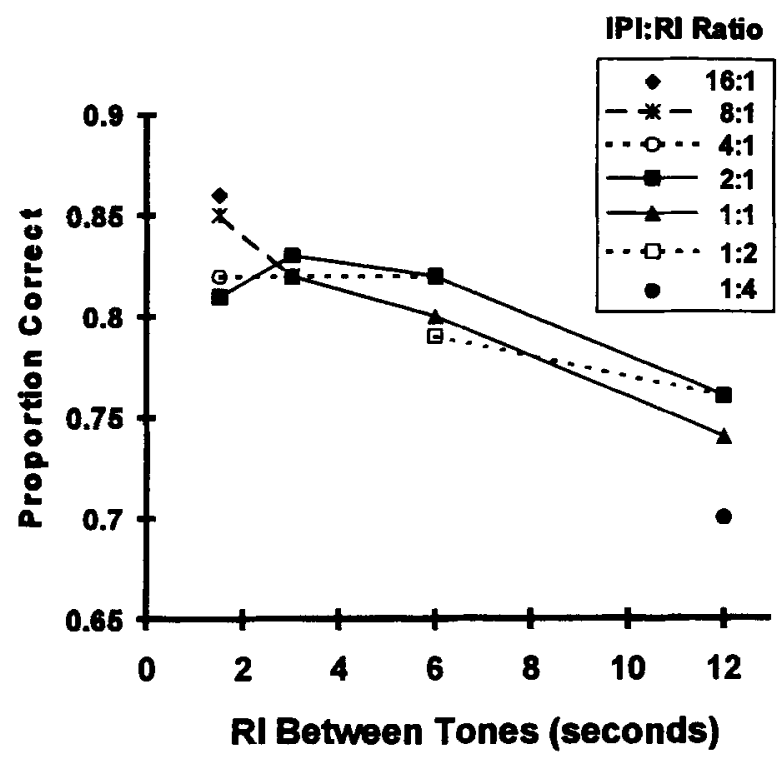

Figure 1. Mean performance level for each combination of the interpair interval (IPI) and the retention interval (RI) between tones to be compared. Means with the same IPI:RI ratio have a common symbol and are connected by lines, as shown in the legend.

1985). The pattern of responses appeared basically similar in ascending and descending trials.

The IPI effect can be attributed to the importance of maintaining temporal distance from previous trials in order to limit proactive interference. The effect of the RI is, however, ambiguous. It could be attributed to the importance of limiting the absolute time between tones or to the importance of having this time be short relative to the distance between the present trial and previous ones.

Additional information about the RI effect is obtained by examining trials with a fixed ratio between the preceding IPI and the RI. For example, a 2:1 ratio was represented in our experiment by IPI:RI combinations of $3: 1.5,6: 3,12: 6$, and $24: 12 \mathrm{sec}$. The other ratios we could examine (with the number of relevant data means shown in parentheses) are 16:1 (1), 8:1 (2), 4:1 (3), 2:1 (4), 1:1 (3), 1:2 (2), and 1:4 (1). All of these means are shown in Figure 1, with lines connecting means with the same IPI:RI ratio. The figure indicates that there was a decline in performance levels across RIs even with the IPI:RI ratio held constant. These effects of absolute time were verified statistically in one-way analyses for data with a particular fixed IPI:RI ratio, with RI as the independent variable. Significant effects of RI emerged in the analyses for an IPI:RI ratio of 2:1 $\left[F(3,81)=3.08, M S_{\mathrm{e}}=\right.$ $0.008, p=.032]$ and of $1: 1\left[F(2,54)=4.83, M S_{\mathrm{e}}=0.009\right.$, $p=.012]$. These are the only two ratios that include at least three data means and also data for the $12-\mathrm{sec}$ RI, where the most extreme forgetting appeared to occur. No significant effect was obtained for the other ratios with two or more data points $(4: 1,8: 1$, and $1: 2)$. However, only one of these ratios (1:2) included a condition with a 12sec RI. 
Inasmuch as a silent distracting task was carried out during the IPI but not during the RI, the IPI effects may be attenuated; the alternation between the distracting task and the tone-memory task could serve as a supplementary, nontemporal basis of distinctiveness separating the trials. However, we see no way in which this could compromise the conclusion that the RI had an additional effect not due to distinctiveness.

\section{DISCUSSION}

Of the vast literature on tone frequency, this is, to our knowledge, the first study to examine effects of time with an attempt to hold distinctiveness constant. The findings clearly suggest that there was an effect of the absolute time that elapsed between tones to be compared, even with the ratio between the IPI (before a trial) and the RI (between the tones) held constant. In addition, there was an effect of temporal distinctiveness as reflected by the IPI.

The RI effects will not surprise those who study event-related, electrical, and magnetoencephalographic brain correlates of memory. Two relevant components (reviewed by Näätänen, 1992) are the "mismatch negativity" response to discriminable changes in a repeated sound and habituation of the "N100" response to new acoustic elements. Both of these phenomena can occur even if the sounds are ignored, but they occur only if the sounds are presented fairly close together, with interstimulus intervals of about $10 \mathrm{sec}$ or less (Böttcher-Gandor \& Ullsperger, 1992; Lu, Williamson, \& Kaufman, 1992; Samms, Hari, Rif, \& Knuutila, 1993). Thus, there appear to be two processes in the brain that depend on the vividness of temporary memory traces. What is critical about these physiological indices is that they are not contaminated by distinctiveness effects like the behavioral measures because, in most such studies, the interval between sounds was held constant throughout a recording session, making the ratio of any two adjacent intersound intervals always $1: 1$.

\section{The Concept(s) of Memory Decay}

The importance of an absolute amount of time in forgetting is usually stated in terms of the concept of "memory decay." Unfortunately, this term is ambiguous enough to be confusing. We will describe three different definitions of memory decay, each one stronger than the previous one, and will describe our position on each one.

Decay Definition 1. The first definition of memory decay for a particular target stimulus is that the ability to recall the target is lost across a certain retention interval at a certain minimum rate regardless of what came before the target, and regardless of whether there is a stimulus or task in the retention interval. Under this definition, however, if decay occurs, it does not rule out the possibility that interference from a stimulus or task in the retention interval can exacerbate the rate at which forgetting occurs, in which case the observed rate of forgetting would overestimate the rate of decay. Under this definition, the present finding of forgetting across RIs that did not contain any task or stimulus, despite a fixed IPI:RI ratio, appears to serve as evidence of decay of memory for tone pitch.

One temporal distinctiveness-based theory of immediate recall, that of Glenberg and Swanson (1986), probably could be adapted to account for our results, but we wonder whether it might implicitly incorporate decay according to the definition offered above, rather than serving as an alternative to decay. In their model, which was designed to account for the free recall of verbal lists, there are "search sets" that become longer in duration as one goes farther back in the list. A search set must be used to retrieve items, and it is more difficult to do so when a larger number of items fall into the same search set. The "ratio rule" emerges from the direct relation between the closeness of an item to the beginning of the list and the temporal extent of the search set that includes it. However, there are two parameters in the model that can lead to a discrepancy between the ratio rule and the predicted results. First, there is a noise parameter that is proportional to the temporal extent of the search set, resulting in poorer performance for more extensive search sets even at constant ratios. Second, there is a temporal param- eter (termed $w 1$ ), such that the subject is able to reinstate the temporal context of the most recent $w 1$ seconds of the list.

Applying Glenberg and Swanson's (1986) theory to our tone-comparison procedure, the relevant "list" would be a series of trials and the relevant "search set" would be one that included the first tone in a pair but excluded tones from previous trials. The decline in performance across RIs with an equal IPI:RI ratio could be accounted for on the grounds that larger intervals contain more noise. Alternatively, it could be accounted for on the grounds that the context that can be reinstated lasts for $w 1$ seconds, shorter than the longest (12-sec) Rl in our study. However, notice that both the noise parameter and the $w 1$ parameter were said to depend on absolute, rather than relative, amounts of time. Given that we required no task during the RI between tones, any noise would be primarily internal, neural noise. The above definition of decay allows that internal noise could be the cause of decay. Similarly, why might $w 1$ allow a reinstatement of the original context of the experiment? One plausible reason is that subjects can make use of a type of memory representation that decays after $w 1$ seconds.

Thus, our empirical result does reveal a departure from the ratio rule. That departure would not have been expected according to many theoretical treatments of the distinctiveness theory (e.g., Bjork \& Whitten, 1974; Crowder, 1976). Although it might be predicted by Glenberg and Swanson (1986), one could argue that theirs is not purely a distinctiveness-based theory and may implicitly model decay.

Our finding of decay according to the definition offered above may apply only to auditory sensory memory. It is not yet clear if it occurs also in verbal short-term memory. Certain other investigators of verbal shortterm memory have observed memory loss over time by using innovative procedures to make sure that it was not a result of retroactive interference during the retention interval (e.g., Reitman, 1974; Wingfield \& Byrnes, 1972). However, these studies cannot resolve the issue, because the ratio of the intertrial interval to the retention interval was not controlled.

Decay Definition 2. The next definition of decay incorporates the postulates of the first one but further states that the decay is not caused merely by interference from some controllable mental process during the retention interval. Any process that can be altered with instructions (e.g., repeating a word; remembering a melody) is considered controllable, as would be particular thoughts that the subject had during the retention interval but could have avoided if asked to. However, this definition leaves open the possibility that the source of decay is some uncontrollable process, such as corrosion of the temporary memory representation through an ambient level of neural noise or depletion of certain physiological resources used up by the temporary memory process. After all, the passage of time logically can have an effect onty through some mediating process.

Obviously, empirical investigation of this definition of decay will be more difficult. In one relevant tone-comparison study, Keller, Cowan, and Saults (1995) found that there was a relatively small (about $10 \%$ ) effect of requiring performance of a distracting, silent mental activity (rehearsing a melody or a verbal sequence) during 10-sec RIs, in comparison with a condition in which the subject was free to think about the standard tone during the RIs. This suggests that there was some effect of controllable mental processes even in the absence of interfering tone stimuli. However, there was considerable memory loss even with no distracting task, holding open the possibility that this stronger type of memory decay exists at least in the sensory domain.

Decay Definition 3. This strongest possible definition of memory decay states that the loss of information occurs according to a fixed rate that is in no way altered by intervening stimuli, tasks, or controllable mental processes. No theory known to us strictly adheres to this definition. The description of the phonological loop provided by Baddeley (1986) comes close in that it states an approximately $2-\mathrm{sec}$ period for phonological memory loss without mentioning potential effects of interference. However, the account does maintain that rehearsal can renew the memory trace. Moreover, we suspect that the lack of emphasis on interference effects was meant as a convenient simplification rather than as a complete description of processing. Cowan et al. (in press) and Neath and Nairne (1995) discuss the potential for interference factors within the word-length effect.

In sum, an extension of theorization from verbal working memory to the domain of auditory memory has proved useful in allowing a 
demonstration that both temporal distinctiveness and the passage of time per se are important in tone memory. It remains to be determined if delay effects can be observed for verbal stimuli with the temporal distinctiveness of trials controlled as in the present study for tones. Questions of the nature of forgetting over time, the existence of memory decay, and its appropriate definition seem fundamental to an understanding of various types of immediate memory and should be the objects of further study in the near future.

\section{REFERENCES}

Baddeley, A. D. (1986). Working memory. Oxford: Oxford University Press, Clarendon Press.

BJORK, R. A., \& WhitTEN, W. B. (1974). Recency-sensitive retrieval processes in long-term free recall. Cognitive Psychology, 6, 173189.

BöTTCHER-GandoR, C., \& UlLSPERgER, P. (1992). Mismatch negativity in event-related potentials to auditory stimuli as a function of varying interstimulus interval. Psychophysiology, 29, 546-550.

Broadbent, D. E. (1958). Perception and communication. London: Pergamon Press.

Brown, G. D. A., \& Hulme, C. (1995). Modeling item length effects in memory span: No rehearsal needed? Journal of Memory \& Language, 34, 594-621.

Cowan, N. (1984). On short and long auditory stores. Psychological Bulletin, 96, 341-370.

CowAN, N. (1988). Evolving conceptions of memory storage, selective attention, and their mutual constraints within the human information processing system. Psychological Bulletin, 104, 163-191.

CowAN, N. (1995). Attention and memory: An integrated framework (Oxford Psychology Series, No. 26). New York: Oxford University Press.

Cowan, N., Day, L., Saults, J. S., Keller, T. A., Johnson, T., \& Flores,

L. (1992). The role of verbal output time in the effects of word length on immediate memory. Journal of Memory \& Language, 31, 1-17.

Cowan, N., Wood, N. L., Nugent, L. D., \& Treisman, M. (in press). There are two word length effects in verbal short-term memory: Opposed effects of duration and complexity. Psychological Science.

Crowder, R. G. (1976). Principles of learning and memory. Hillsdale, NJ: Erlbaum.

CROWDER, R. G. (1993). Short-term memory: Where do we stand? Memory \& Cognition, 21, 142-145.

Elliott, P. B. (1964). Tables of $d^{\prime}$. In J. A. Swets (Ed.), Signal detection and recognition by human observers (pp. 651-684). New York: Wiley

GARDINER, J. M., \& GREGG, V. H. (1979). When auditory memory is not overwritten. Journal of Verbal Learning \& Verbal Behavior, 18 705-719.

Glenberg, A. M., \& SWanson, N. C. (1986). A temporal distinctiveness theory of recency and modality effects. Journal of Experimental Psychology: Learning, Memory, \& Cognition, 12, 3-15.

Green, D. M. (1964). Psychoacoustics and detection theory. In J. A. Swets (Ed.), Signal detection and recognition by human observers (pp. 58-94). New York: Wiley.
HeLlström, Å. (1985). The time-order error and its relatives: Mirrors of cognitive processes in comparing. Psychological Bulletin, 97, 35-61.

Keller, T. A., Cowan, N., \& Saults, J. S. (1995). Can auditory memory for tone pitch be rehearsed? Journal of Experimental Psychology: Learning, Memory, \& Cognition, 21, 635-645.

KePPEL, G., \& UNDERWOOD, B. J. (1962). Proactive inhibition in shortterm retention of single items. Journal of Verbal Learning \& Verbal Behavior, 1, 153-161.

KoppenAal, L., \& Glanzer, M. (1990). An examination of the continuous distractor task and the "long-term recency effect." Memory \& Cognition, 18, 183-195.

LoFTus, G. R., \& Masson, M. E. J. (1994). Using confidence intervals in within-subject designs. Psychonomic Bulletin \& Review, 1, 476490.

Lu, Z.-L., Williamson, S. J., \& Kaufman, L. (1992). Physiological measures predict behavioral lifetime of human auditory sensory memory. Science, 258, 1668-1670.

MASSARO, D. W. (1970). Retroactive interference in short-term recognition memory for pitch. Journal of Experimental Psychology, 83, 32-39.

MCGEOCH, J. A. (1932). Forgetting and the law of disuse. Psychological Review, 39, 352-370

MeLton, A. W. (1963). Implications of short-term memory for a general theory of memory. Journal of Verbal Learning \& Verbal Behavior, 2, 1-21.

MiLLER, G. A. (1956). The magical number seven, plus or minus two: Some limits on our capacity for processing information. Psychological Review, 63, 81-97.

NÄÄTÄNEN, R. (1992). Attention and brain function. Hillsdale, NJ: Errlbaum.

NAIRNE, J. S. (1992). The loss of positional certainty in long-term memory. Psychological Science, 3, 199-202.

NEATH, I., \& NAIRNE, J. S. (1995). Word-length effects in immediate memory: Overwriting trace decay theory. Psychonomic Bulletin \& Review, 2, 429-441.

Peterson, L. R., \& Peterson, M. J. (1959). Short-term retention of individual verbal items. Journal of Experimental Psychology, 58, 193-198.

REITMAN, J. S. (1974). Without surreptitious rehearsal, information in short-term memory decays. Journal of Verbal Learning \& Verbal Behavior, 13, 365-377.

Samms, M., Hari, R., Rif, J., \& KnuUtila, J. (1993). The human auditory sensory memory trace persists about $10 \mathrm{sec}$ : Neuromagnetic evidence. Journal of Cognitive Neuroscience, 5, 363-370.

SCHWEICKERT, R., \& BORUFF, B. (1986). Short-term memory capacity: Magic number or magic spell? Journal of Experimental Psychology: Learning, Memory, \& Cognition, 12, 419-425.

WingField, A., \& BYrNes, D. L. (1972). Decay of information in short-term memory, Science, 176, 690-692.

(Manuscript received November 4, 1996 revision accepted for publication February 24, 1997.) 